\title{
A New Two-Stage Bargaining Game Approach for Intra- and Inter-WBAN Management
}

\author{
Sungwook Kim \\ Department of Computer Science, Sogang University, 35 Baekbeom-ro (Sinsu-dong) Mapo-gu, Seoul 04107, Republic of Korea \\ Correspondence should be addressed to Sungwook Kim; swkim01@sogang.ac.kr
}

Received 2 September 2021; Revised 4 November 2021; Accepted 15 November 2021; Published 27 November 2021

Academic Editor: Alessandro Bazzi

Copyright ( 92021 Sungwook Kim. This is an open access article distributed under the Creative Commons Attribution License, which permits unrestricted use, distribution, and reproduction in any medium, provided the original work is properly cited.

\begin{abstract}
The Internet of Medical Things (IoMT) is an amalgamation of smart devices to operate the wireless body area network (WBAN) by using networking technologies. To reduce the burden on WBANs, they link to the mobile edge computing (MEC), on which captured medical data can be stored and analyzed. In this paper, we design a new control scheme to effectively share the limited computation and communication resources in the MEC-assisted WBAN (M-W) platform. Based on the bargaining game theory, our proposed scheme explores the mutual benefits of intra- and inter-WBAN interactions. To dynamically adapt the current system conditions, we shape each WBAN's aspirations to reach a reciprocal consensus for different application services. Utilizing two control factors, we provide a unifying framework for the study of intra- and inter-WBAN bargaining problems to share the limited system resource. Based on the feasibility and real-time effectiveness, the main novelty of the proposed scheme is the ability to achieve a relevant tradeoff between efficiency and fairness through the interactive bargaining process. At last, the experimental results show that the proposed scheme achieves substantial performance improvements to the comparison schemes.
\end{abstract}

\section{Introduction}

With the widespread application of the Internet of Things (IoT), a large number of smart devices are connected via Internet for ubiquitous services. It has been envisioned as one of the most promising networking paradigms that bridge the gap between the cyber and physical world. In recent years, the rise of the IoT paradigm is leading to a paradigm shift in all the areas of human-machine interaction. As predicted by Ericsson and Cisco Inc., more than 50 billion devices will connect to Internet by the year 2025, and the data produced by IoT devices will exceed 500 zettabytes (ZB). Most of these devices are geographically distributed in order to provide collaboratively variable and flexible communication, computation, and storage services. Thanks to the advanced computation and communication technologies, the IoT paradigm can change many aspects of both traditional industrial productions and our everyday living $[1,2]$.

Healthcare in modern days has been transformed by advances in the IoT technology; the common practice of clinical treatment is gradually being overhauled by ubiquitous healthcare systems. Therefore, medical practices need to respond to these undergoing crucial changes by developing strategies to provide the best patient care while remaining profitable. The wireless body area network (WBAN) has emerged as a key technology to access healthcare services anytime and anywhere. It is a kind of wireless communication networks centered on the human body, which can collect physiological, behavioral, and other health-related data in real time. Therefore, the WBAN is capable of providing real-time e-healthcare services to patients with emergency medical needs in a cost-effective and reliable manner. As a significant component of the IoT, the WBAN is known as Internet of Medical Things (IoMT) and influences the human by human health monitoring through wearable or implanted medical sensors [2-5].

Usually, WBANs generally have tiny, lightweight, wireless IoMT devices that are located in, on, or around human bodies. Based on one-hop and two-hop star topology in physical and data link layers, these devices are able to monitor the functions of the human body and surrounding 
features. As the latest international standard for WBANs, the IEEE 802.15.6 standard has been proposed for specified service requirements in short-range communications among IoMT devices. It aims to provide a protocol for low-power, short-range, and extremely reliable wireless communications within the surrounding area of the human body, supporting a vast range of data rates for different applications. Therefore, all solutions to be proposed for the WBAN should be based on the 802.15.6 standard to address different priorities and specifications of WBANs. Specifically, the IEEE 802.15.6 based WBSN scheme consists of one coordinator node, which is called local processing unit, and several IoMT devices. The coordinator node uses beacon mode with super-frame structure and duty cycle mechanism to reduce energy consumption. Therefore, another goal of the IEEE 802.15.6 was to define new physical and medium access control layers for WBANs $[6,7]$.

In parallel, the advancement of IoMT brings some challenges to its implementation. Most of the IoMT devices are embedded with low processor and storage capacity, and they have limited resources for computing, storage, and communication. Therefore, the computing capacity improvement of IoMT devices is one of the major technical challenges in handling computation-intensive medical applications. Recently, the proliferation of mobile edge computing (MEC) is conceived as a favorable solution to tackle such a challenge. Originally, the basic idea behind MEC is that by background running of related processing tasks, applications perform better. By offloading the medical analysis task to the MEC server in proximity, the burden of IoMT devices can be released while providing sufficient computation resources. Practically, it is worth noticing that the combination of MEC and WBAN enables flexible and rapid deployment of new IoMT applications and services [5].

To implement the MEC-assisted WBAN (M-W) system, it is necessity to develop an effective control algorithm that shares the limited system resource in a fair-efficient manner. With the wide spread and application of WBANs, massive health-related data access and different service requirements pose intra- and inter-WBAN resource allocation problems. Usually, implanted IoMT devices are associated with the limited computing capabilities. However, computation-intensive WBAN applications require high processing and energy resources, which might eventually impact on a mission success. To alleviate the restriction of computing power in WBANs, each individual WBAN decides whether to perform the medical analysis task by local computing or offload it to the MEC server. In addition, for the environment of urban $\mathrm{M}-\mathrm{W}$ platform, the spectrum resource of MEC is scarce when there are numerous WBANs. Therefore, it should be efficiently shared among heterogeneous WBANs $[4,5,8]$.

To effectively share the intra-WBAN computation and inter-WBAN communication resources, one of the key challenges is to understand the behaviors of self-regarding IoMT devices while dynamically negotiating conflicting requests. To handle this challenge, we adopt the bargaining game theory to guide these selfish IoMT devices to achieve a globally desirable $\mathrm{M}-\mathrm{W}$ system performance. As a branch of game theory, the bargaining game theory deals with the analysis of sharing problems, in which some game players bargain over the division of limited resources. Usually, the bargaining theory has been a central research topic in economics for over seven decades and, in recent years, it has become an interesting issue in the network resource management. For the M-W system, this paper proposes a logicbased bargaining solution by considering the interaction of IoMT applications and the ordinal structure of their preferences [9].

1.1. Technical Concepts. In 1975, the well-known Kalai-Smorodinsky bargaining solution (KSBS) for $n$-person bargaining problems captures a simple intuition. To implement this solution, the disagreement point, representing the outcome that will prevail when the players do not cooperate, and the utopia point, representing an ideal outcome in which each player is awarded the maximum payoff, are proposed. Starting from the disagreement point, increase the surplus allocated to every player in the direction of the utopia point, increasing each player's outcome in a proportional way. Since 1975, a plethora of bargaining studies argued that another point, called reference point, is necessary to coordinate the bargaining behavior and outcomes. The reference point idea stipulates that individual players evaluate outcomes with respect to a reference outcome in many decision making situations. Therefore, it can be seen as allocating losses with respect to utopia point or as allocating gains from the reference point in the direction of utopia point [10].

The way disagreement and reference points are incorporated into most bargaining models implies that they may have an indirect influence on the negotiated agreement through their influence on the best case scenario, i.e., aspiration. In the KSBS, players' aspirations are directly derived from the disagreement point, which represents the worst case scenario, i.e., anchor. The Gupta and Livne bargaining solution (GLBS) employs the reference point as an anchor and the ideal point as an aspiration. In the tempered aspirations bargaining solution (TABS), aspiration is derived from the reference point instead of the disagreement point. Therefore, the TABS employs the disagreement point as an anchor and the tempered ideal point as an aspiration. If the reference point completely determines both the anchor and aspiration, this solution is called the local Kalai-Smorodinsky bargaining solution (LKSBS) $[11,12]$.

Recently, the anchor and aspiration bargaining solution $(A A B S)$ has been introduced based on what would happen in the best case scenario, i.e., aspiration, and in the worst case scenario, i.e., anchor, in negotiations. The basic idea of $A A B S$ is to capture the influence of the reference point on the anchor and its influence on players' aspirations, respectively. By using two control parameters, the $A A B S$ understands a bargaining solution as the representation of arbitrator's distributive preference. Under this interpretation, two parameters can be considered as the arbitrator's opinion on how effective the reference and disagreement points should 
be in reaching a settlement in a given situation. Generally speaking, the existing characterizations in the KSBS, GLBS, $T A B S$, and $L K S B S$ can be obtained as special cases of the $A A B S$ [11].

1.2. Main Contributions. According to the bargaining solutions, we can effectively solve the resource allocation problems in the M-W system. By considering the different IoMT applications, we design an intra-WBAN bargaining game model to offload its computing tasks to the MEC. Then, an inter-WBAN bargaining game model is developed to share the limited spectrum resource in the M-W system. For this dual bargaining process, the ideas of $A A B S$ and $K S B S$ are adopted based on the service characteristics. During the step-by-step bargaining iteration, our proposed scheme appropriately handles the growing demand of WBAN service requests while leading to an appropriate system performance balance. In detail, the major contributions of this study are summarized as follows:

(i) We investigate the MEC-assisted WBAN infrastructure, its requirements, and its relevance. Then, we adopt the concepts of AABS and KSBS to formulate our two-stage bargaining game for the $\mathrm{M}-\mathrm{W}$ system.

(ii) At the first stage, the intra-WBAN bargaining process is designed by using the $A A B S$. It can coordinate the offloading computation and local computation in each individual WBAN.

(iii) At the second stage, the inter-WBAN bargaining process is developed based on the KSBS. It distributes the MEC spectrum resource for multiple WBANs by considering the features of heterogeneous IoMT services.

(iv) Based on the iterative combination of intra- and inter-WBAN bargaining processes, we can compromise the conflicting IoMT service requirements while ensuring a globally desirable solution.

(v) Through the simulation analysis, the performance of our proposed scheme is demonstrated by comparison with three relevant WBAN protocols. The comparison results validate the effectiveness of our two-stage bargaining approach while leveraging a mutual advantage agreement for conflicting control issues.

1.3. Organization. The remainder of the article is organized as follows. In Section 2, we discuss the related work relevant to this paper. Section 3 presents the M-W system platform and the background knowledge and fundamental ideas of $A A B S$ and $K S B S$. Then, we explain in detail our two-stage bargaining game model and provide the main steps of our proposed algorithm in detail. In Section 4, we show the performance of the proposed scheme by comparing it with other existing state-of-the-art protocols. Finally, Section 5 concludes this paper while mentioning few future research directions.

\section{Related Work}

Enormous previous research has investigated the issues of IoMT paradigm. This section presents a brief survey in the area of recent WBAN control protocols. In [4], Yuan et al. propose the Two-level Game based Computation Offloading (TGCO) scheme to optimize the WBAN resource allocation problem. First, they define a utility function to quantify the impact of resource allocation and make offload decisions according to the task characteristics. Second, the utility maximization problem is constructed based on the game theory. They adopt a noncooperative potential game model to simplify the utility maximization problem without tedious verification of the existence of equilibrium solution. Finally, a novel two-level optimization algorithm is developed to solve the problem in MEC service scenarios. In the two levels, the offloading and unloading decisions are made to allocate different local and server computing resources to tasks of certain WBANs. Finally, performance evaluation results show that the TGCO scheme can improve the average processing delay, energy consumption, and network utility at different data arrival rates [4].

The Edge Computing based Health Monitoring (ECHM) scheme is a new MEC-enabled health monitoring algorithm for IoMT services [5]. A major challenge of this scheme is to minimize the IoMT system cost, which depends on the medical criticality and energy consumption of health monitoring packets. In the ECHM scheme, the IoMT is divided into two subnetworks, i.e., intra-WBANs and beyond-WBANs. For the intra-WBAN, a gateway regulates the transmission rates of body sensors and attempts to minimize the cost of individual patients based on the cooperative game model. For the beyond-WBAN, a noncooperative potential game model is designed to coordinate the medical information analysis between local devices and the edge server. Considering individual rationality and potential selfishness, the potential game obtains the strategy profile that can reach the Nash equilibrium in a decentralized manner. Performance analysis shows the effectiveness of the ECHM scheme with the benefit from MEC [5].

In [8], the Individual Differentiated Coexisting Mechanism (IDCM) scheme is proposed to deal with the multiple WBANs. The IDCM scheme consists of four algorithms; (i) time slot allocation, (ii) access control, (iii) active part interleaving, and (iv) power control. These algorithms can guarantee the transmission performance of each WBAN while providing the system resource for each WBAN depending on its own importance and service requirements. For the timeslot allocation algorithm, a game based approach is proposed to deal with timeslot allocation problem among multiple WBANs. Access control and active period scheduling algorithms are designed based on the IEEE 802.15.6 standard protocol. For the power control algorithm, a mobility prediction is considered to deal with the transmission power of the WBANs. The simulation results demonstrate that the IDCM scheme is stable to provide the WBAN efficiency [8].

Until now, some WBAN protocols have been proposed with novel ideas for the IoMT paradigm. However, none of 
research literature considers the dual bargaining game approach from a coordinated viewpoint. Due to the desirable characteristics of game theory, our two-stage integrated bargaining method can get a globally desirable M-W system performance for the WBAN resource sharing problem.

\section{The Proposed M-W System Resource Sharing Scheme}

In this section, the M-W system platform and operation scenario are introduced. Then, the proposed two-stage bargaining game is formulated based on the $A A B S$ and $K S B S$ to share the M-W system resource. Finally, the main step procedures of our proposed scheme are described.

3.1. M-W System Infrastructure and Dual Bargaining Game Model. In case of modern ubiquitous healthcare, human customers are equipped with IoMT-assisted WBANs, for real-time monitoring of physiological parameters. In this study, we assume a scenario of multiple WBANs with one MEC server to provide health monitoring services. The MEC server $(\mathscr{M})$ is a mobility-enhanced small-scale cloud server that is located at the edge of the Internet. The $\mathscr{M}$ can provide powerful computing resources to WBANs with lower latency. Let there be $n$ number of WBANs, which are represented as a set $\mathfrak{B}=\left\{\mathscr{B}_{1}, \ldots, \mathscr{B}_{n}\right\}$. In each individual WBAN, there are number of heterogeneous IoMT devices, which are wearable or implanted inside the human body, and one local processing unit (LPU), which serves the WBAN data aggregation and asks the $\mathscr{M}$ for both communication and computation resources. Therefore, multiple LPUs can offload several tasks to the $\mathscr{M}$ server based on the current $\mathrm{M}-\mathrm{W}$ system condition. The overview of $\mathrm{M}-\mathrm{W}$ system platform is shown in Figure $1[13,14]$.

Our two-stage bargaining game model consists of intraand inter-WBAN bargaining processes to share the M-W system communication and computation resources. In the intra-WBAN bargaining process, each LPU receives periodically the health information from its corresponding IoMT devices. According to its limited computation capacity, the LPU decides whether to perform the medical analysis task by local computing or offload it to the $\mathscr{M}$. In a parallel and distributed manner, $n$ LPUs act independently and make their offload decisions for their corresponding WBANs. In the inter-WBAN bargaining process, we are mainly concerned with the spectrum allocation problem among WBANs, which contend for the $\mathscr{U}$ 's spectrum resource. To share optimally the limited communication and computation resources of $\mathrm{M}-\mathrm{W}$ system, the intra- and interWBAN bargaining processes work together toward an appropriate system performance. Formally, we define our twostage bargaining game entities, i.e., $\mathbb{G}=\left\{\mathbb{G}_{1 \leq i \leq n}^{I}, \mathbb{G}^{I I}\right\}=$ $\left\{\left\{\mathbb{G}_{i}^{I} \mid \mathscr{L}_{i}, \mathfrak{P}_{\mathscr{L}}, \mathcal{S}_{i}, \quad\left(\mathcal{S}_{i}^{L}, U_{i}^{L}\right),\left(\mathcal{S}_{i}^{O}, U_{i}^{O}\right)\right\}, \mathscr{M},\left\{\mathbb{G}^{I I} \mid \mathfrak{B}, \mathfrak{M}\right.\right.$, $\left.\left.\mathfrak{U}_{\mathscr{B}_{i} \in \mathfrak{B}}, N_{\mathscr{B}_{i}}\right\}, T\right\}$, and Table 1 lists the notations used in this paper.

(i) In the two-stage game $\mathbb{G}, \mathbb{G}_{i}^{I}$ is the intra-WBAN bargaining game for the $\mathscr{B}_{i}$, and $\mathbb{G}^{I I}$ is the interWBAN bargaining game for the M-W system. $\mathbb{G}_{i}^{I}$ and $\mathbb{G}^{I I}$ games are mutually and reciprocally interdependent in an interactive manner.

(ii) In the $\mathbb{G}_{i}^{I}, \mathscr{L}_{i}$ is the $\mathscr{B}_{i}$ 's LPU and $\mathfrak{P}_{\mathscr{L}}$ is the total computation capacity of $\mathscr{L}_{i}$.

(iii) $\mathcal{S}_{i}$ is the current computation workload in the $\mathscr{B}_{i}$. The $\mathcal{S}_{i}$ is divided into two parts, i.e., $\mathcal{S}_{i}^{L}$ and $\mathcal{S}_{i}^{O}$, for the local and offload computing workloads, where $\mathcal{S}_{i}=\mathcal{S}_{i}^{L}+\mathcal{S}_{i}^{O}$.

(iv) In the $\mathbb{G}_{i}^{I}$, the $\mathcal{S}_{i}^{L}$ and $\mathcal{S}_{i}^{O}$ are game players. Therefore, the $\mathbb{G}_{i}^{I}$ is a two-player bargaining game.

(v) $U_{i}^{L}$ is the utility function of local computing workload $\left(\delta_{i}^{L}\right)$, and $U_{i}^{O}$ is the utility function of offload computing workload $\left(\delta_{i}^{O}\right)$.

(vi) $\mathscr{M}$ is a MEC server for the $\mathscr{B}_{1 \leq i \leq n}$, and $\mathfrak{B}$ is the set of WBANs, where $\mathscr{B}_{i} \in \mathfrak{B}$. $\mathfrak{M}$ is the total spectrum resource of $\mathscr{M}$.

(vii) In the $\mathbb{G}^{I I}$, the $\mathscr{B}_{1 \leq i \leq n}$ are game players, and $\mathfrak{U}_{\mathscr{B}_{i}}$ is the utility function of $\mathscr{B}_{i}$. Therefore, the $\mathbb{G}^{I I}$ is a $n$-player bargaining game.

(viii) Each application of IoMT devices has its own preference level, where $l_{m}, l_{M}$ are the minimum and maximum preference levels, respectively. The $N_{\mathscr{B}_{i}}$ is the set of $\mathscr{B}_{i}{ }^{\prime}$ running applications' preferences.

(ix) $T=\left\{t_{1}, \ldots, t_{c}, t_{c+1}, \ldots,\right\}$ denotes time period, which is represented by a sequence of time steps.

3.2. The Basic Idea and Fundamental Concept of AABS. To characterize the basic concepts of bargaining solutions, we assume an $n$-player bargaining problem. Let $S, d$, and $r$ be the set of feasible outcomes, disagreement point, and reference point, respectively, where $S \subset \mathbb{R}^{n}, r \geq d$, and $d, r \in \mathrm{S}$. Simply, a bargaining problem is denoted by $(S, d, r)$, and let $\sum^{n}$ be the family of all $n$-player bargaining problems. For every $x=\left(x_{1}, \ldots, x_{n}\right) \in S$ with $x>d$, let $A(S, x)$ be the aspiration vector such that $A_{i}(S, x) \equiv \max \left\{t \in \mathbb{R} \mid\left(t, x_{-i}\right) \in S\right\} \quad$ where $x_{-i}=\left(x_{1}, \ldots, x_{i-1}, x_{i+1}, \ldots, x_{n}\right)$. Accordingly, $A(S, d)$ is the ideal point and $A(S, r)$ is the tempered aspirations point. A bargaining solution concept for $(S, d, r)$ is a function $f: \sum^{n} \longrightarrow \mathbb{R}^{n}$ where $(S, d, r) \in \sum^{n}$. By using the two control factors, i.e., $0 \leq \delta_{I}, \delta_{I I} \leq 1$, we can define the $A A B S$, where $\delta_{I}$ can be interpreted as the power of $r$ in determining the anchor and $\delta_{I I}$ can be interpreted as the influence of $r$ in shaping players' aspiration. For every $(S, d, r) \in \Sigma^{n}$, the $A A B S$ with $\delta_{I}$ and $\delta_{I I}$ values, i.e., $A A B S^{\delta_{I}, \delta_{I I}}(S, d, r)$, is defined as follows [11]:

$$
\begin{aligned}
& A A B S^{\delta_{I}, \delta_{I I}}(S, d, r)=\max _{\lambda \in[0,1]}\left\{\lambda \mid\left(\left[\lambda \times \mathscr{Q}\left(S, \mathscr{X}^{\mathscr{A}}\right)\right]+\left[(1-\lambda) \times \mathscr{X}^{d}\right]\right)\right\}, \\
& \text { s.t. }\left\{\begin{array}{l}
\mathscr{X}^{\mathscr{A}}=\left(\left(\delta_{I I} \times r\right)+\left(\left(1-\delta_{I I}\right) \times d\right)\right), \\
\mathscr{X}^{d}=\left(\left(\delta_{I} \times r\right)+\left(\left(1-\delta_{I}\right) \times d\right)\right), \\
A A B S^{\delta_{I}, \delta_{I I}}(S, d, r) \in S .
\end{array}\right.
\end{aligned}
$$




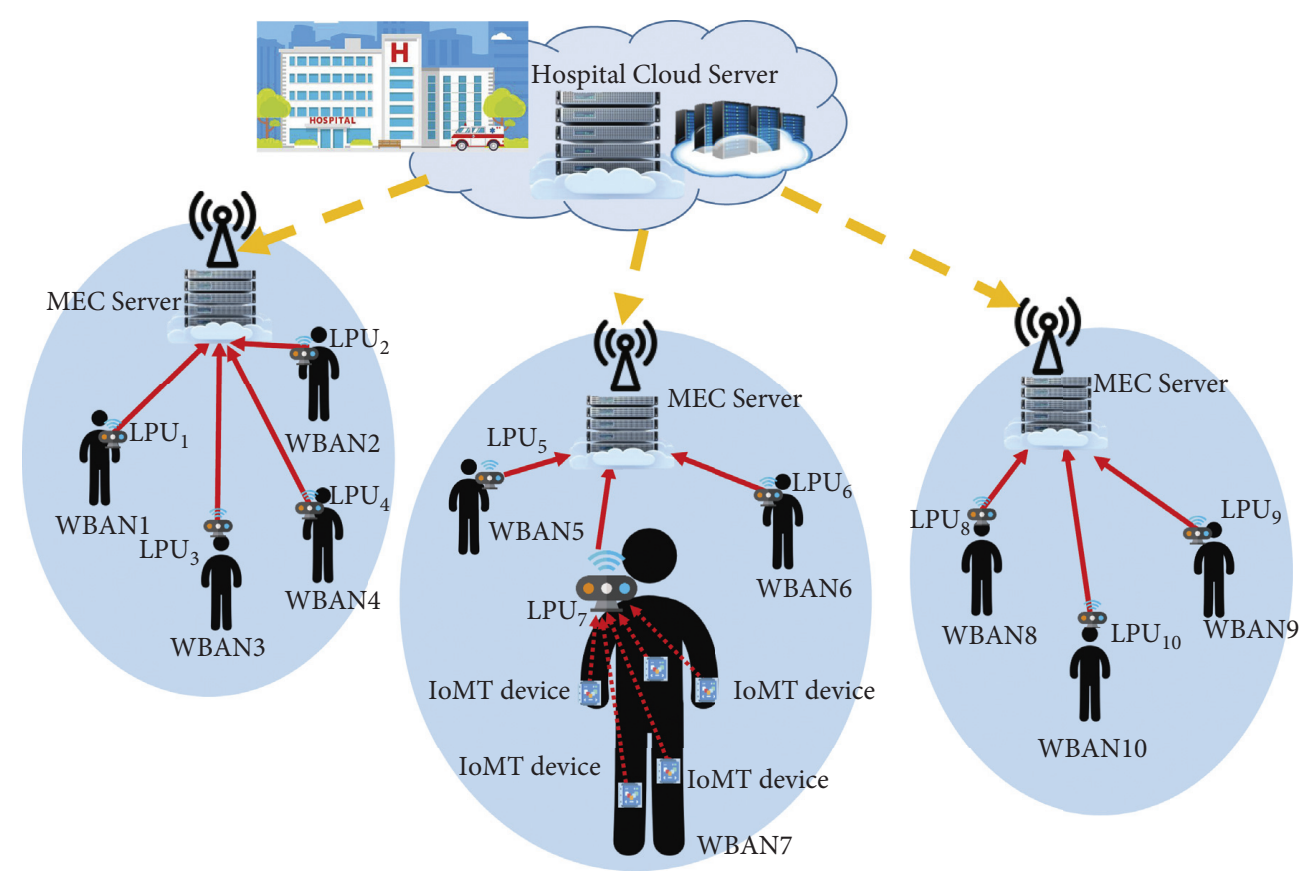

Figure 1: The overview of M-W system platform.

Geometrically, the $A A B S$ proposes the maximum point of the bargaining set on the line segment connecting the aspiration, i.e., $\mathscr{X}^{\mathscr{d}}$, and the anchor, i.e., $\mathscr{X}^{d}$. With $0 \leq \delta_{I}, \delta_{I I} \leq 1$, the collection of all such possible solutions constitutes the available set of AABS. Simply, the KSBS is obtained with $\delta_{I}=0$ and $\delta_{I I}=0$. Therefore, we can think that the KSBS is the maximum point of the bargaining set on the line segment connecting the ideal point and the disagreement point [9]. The ideas of different bargaining solutions are illustrated in Figure 2.

\subsection{The Two-Stage Bargaining Game in the $M-W$ Platform.} At each time $t_{c} \in T$, IoMT devices in $\mathscr{B}_{i}$ generate independently their service data; $\mathcal{S}_{i}\left(t_{c}\right)$ is the $\mathcal{S}_{i}$ at time $t_{c}$. To process the $\mathcal{S}_{i}\left(t_{c}\right)$, the $\mathscr{L}_{i}$ needs an appropriate computing power capability to collect and analyze IoMT data. However, it has a limited computation capacity. To reduce the burden on the $\mathscr{L}_{i}$, the $\mathcal{S}_{i}\left(t_{c}\right)$ can be partially offloaded to its corresponding MEC. In the proposed scheme, we design the $\mathbb{G}_{i}^{I}$ for this computation offloading problem. To effectively exploit the computing capability in MEC while considering the current computation overhead of $\mathscr{L}_{i}$, one major challenge is how to split the $\mathcal{S}_{i}\left(t_{c}\right)$ to proceed the offload service; the $\mathcal{S}_{i}\left(t_{c}\right)$ is divided into two parts, where the $\delta_{i}^{L}\left(t_{c}\right)$ is executed locally by the $\mathscr{L}_{i}$ and $\mathcal{S}_{i}^{O}\left(t_{c}\right)$ is offloaded to the MEC. Therefore, the intra-WBAN data offloading problem is formulated as a two-player, i.e., $\delta_{i}^{L}\left(t_{c}\right)$ and $\mathcal{S}_{i}^{O}\left(t_{c}\right)$, bargaining game. For the local computing service, the utility function of $\mathcal{S}_{i}^{L}(t)$, i.e., $U_{i}^{L}(\cdot)$, is defined according to the current $\mathrm{M}-\mathrm{W}$ system conditions.
TABle 1: The notations for abbreviations, symbols, and parameters.

\begin{tabular}{lc}
\hline Acronym & Explanations \\
\hline IoMT & Internet of Medical Things \\
WBAN & Wireless body area network \\
MEC & Mobile edge computing \\
M-W & MEC-assisted WBAN \\
KSBS & Kalai-Smorodinsky bargaining solution \\
GLBS & Gupta and Livne bargaining solution \\
TABS & Tempered aspirations bargaining solution \\
LKSBS & Local Kalai-Smorodinsky bargaining solution \\
AABS & Anchor and aspiration bargaining solution \\
TGCO & Two-level Game based Computation Offloading \\
ECHM & Edge Computing based Health Monitoring \\
IDCM & Individual Differentiated Coexisting Mechanism \\
LPU & Local processing unit \\
Notations & Explanations \\
$\mathscr{M}$ & MEC server \\
$\mathscr{B}_{i}$ & The $i$ 's WBAN \\
$\mathbb{G}_{i}^{I}$ & The intra-WBAN bargaining game for the $\mathscr{B}_{i}$ \\
$\mathbb{G}^{I I}$ & The inter-WBAN bargaining game for the M-W \\
$\mathscr{L}_{i}$ & The system \\
$\mathfrak{P}_{\mathscr{L}}$ & The $\mathscr{B}_{i}$ 's LPU \\
$\mathcal{S}_{i}^{L}$ & The total computation capacity of $\mathscr{L}_{i}$ \\
$\mathcal{S}_{i}^{O}$ & The local computing workload \\
$U_{i}^{L}$ & The offload computing workload \\
$U_{i}^{O}$ & The utility function of $\mathcal{S}_{i}^{L}$ \\
$\mathfrak{M}^{L}$ & The preferences \\
$\mathfrak{U}_{\mathscr{B}_{i}}$ & $\mathscr{B}_{i}$ \\
$N_{\mathscr{B}_{i}}$ & The sunction of $\mathcal{S}_{i}^{O}$ \\
&
\end{tabular}




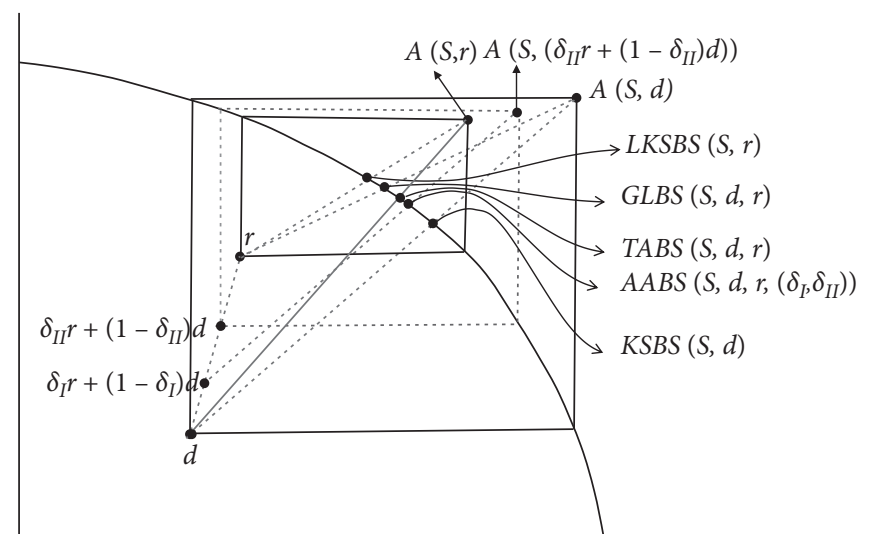

Figure 2: The ideas of different bargaining solutions.

$$
\begin{aligned}
U_{i}^{L}\left(\tau_{i}, \mathcal{S}_{i}\left(t_{c}\right), \mathscr{W}_{\mathscr{M}}^{t_{c-1}}\right)= & {\left[\frac{\mathscr{Z}}{\eta+\exp (\mathscr{Z})} \times \mathscr{H}_{i}^{t_{c}}\right] } \\
& +\log \left(\left(\mathscr{Z} \times\left(1-\mathscr{W}_{\mathscr{M}}^{t_{c-1}}\right)\right)+\theta\right), \\
\text { s.t., } \mathscr{Z}= & \frac{\left(\tau_{i} \times \mathcal{S}_{i}\left(t_{c}\right)\right)}{\mathcal{S}_{i}\left(t_{c}\right)}, \\
\mathscr{H}_{i}^{t}= & \frac{\left(\tau_{i} \times \mathcal{S}_{i}\left(t_{c}\right)\right)}{\mathfrak{P}_{\mathscr{L}}},
\end{aligned}
$$

where $\tau_{i}$ is the task offloading ratio where $0 \leq \tau_{i} \leq 1 . \eta, \theta$ are the adjustment parameters for the $U_{i}^{L}(\cdot)$, and $\mathscr{W}_{\mathscr{M}}^{t_{c-1}}$ is the $\mathscr{M}$ 's spectrum usage at time $t_{c-1}$. For the offload computing service, the utility function of $\mathcal{S}_{i}^{O}\left(t_{c}\right)$, i.e., $U_{i}^{O}(\cdot)$, is also defined according to the current information of $\mathscr{B}_{i}$.

$$
U_{i}^{O}\left(\tau_{i}, \mathcal{S}_{i}\left(t_{c}\right), \mathscr{W}_{\mathscr{M}}^{t_{c-1}}\right)=\exp \left(\frac{\left(\tau_{i} \times \mathcal{S}_{i}\left(t_{c}\right)\right)}{\mathcal{S}_{i}\left(t_{c}\right)} \times\left(\left(1-\mathscr{W}_{\mathscr{M}}^{t_{c-1}}\right) \times \beta\right)\right)-\sigma
$$

where $\beta$ and $\sigma$ are the control parameters for the $U_{i}^{O}(\cdot)$. To adaptively adjust the $\tau_{i}$ value at time $t_{c}$, it is necessary to consider the current computation and communication situations of $\mathscr{B}_{i}$. These two factors are dynamically changeable and equally important for the intra-WBAN bargaining process. Therefore, we adopt the idea of $A A B S$ for the $\mathbb{G}_{i}^{I}$ game, and the reference point at time $t_{c}$, i.e., $r_{i}\left(t_{c}\right)=\left(r_{i}^{L}\left(t_{c}\right), r_{i}^{O}\left(t_{c}\right)\right)$, and the disagreement point at time $t_{c}$, i.e., $d_{i}\left(t_{c}\right)=\left(d_{i}^{L}\left(t_{c}\right), d_{i}^{O}\left(t_{c}\right)\right)$, should be decided in an online fashion. To adapt the current intra-WBAN conditions, we assume that the $r_{i}\left(t_{c}\right)$ decision problem is another bargaining problem. For this problem, the axiom of individual monotonicity is essential for local and offloading computation tasks. Therefore, the concept of KSBS is suitable. The KSBS for the $r_{i}\left(t_{c}\right)$ decision, i.e., $K S B S_{r_{i}}\left(S^{r_{i}}, d\right)$, is obtained with the restricted service request domain, i.e., $S^{r_{i}}=\mathcal{S}_{i}\left(t_{c}\right) / \omega$ where $\omega$ is a diminishing factor. Therefore, the $r_{i}\left(t_{c}\right)$ is given by

$$
r_{i}\left(t_{c}\right)=\operatorname{KSBS}_{r_{i}}\left(S^{r_{i}}, d\right)=\max _{\left(\delta_{i}^{L}\left(t_{c}\right), \mathcal{\delta}_{i}^{O}\left(t_{c}\right)\right) \in\left(S^{\left.r_{i}, d\right)}\right.}\left(\min _{\mathscr{V} \in\left\{\delta_{i}^{L}\left(t_{c}\right), \delta_{i}^{O}\left(t_{c}\right)\right\}} \frac{\mathscr{V}-d_{\mathscr{V}}}{A_{\mathscr{V}}\left(S^{r_{i}}, d\right)-d_{\mathscr{V}}}\right)
$$

In this study, we set $d_{i}\left(t_{c}\right)=(0,0)$ as the result if the $\mathcal{S}_{i}^{L}\left(t_{c}\right)$ and $\mathcal{S}_{i}^{O}\left(t_{c}\right)$ cannot reach an agreement of offloading strategy. With the $r_{i}\left(t_{c}\right)$ and $d_{i}\left(t_{c}\right)$, we also decide the values of $\delta_{I}$ and $\delta_{I I}$ to get the $A A B S$. Under diverse M-W system environments, the values of $\delta_{I}$ and $\delta_{I I}$ should be dynamically modified to improve the effectiveness of $A A B S$. In this study, they are adaptively decided based on the current information of each WBAN. At time $t_{c}$, the $\mathscr{B}_{i}{ }^{\prime}$ s $\delta_{I}$ and $\delta_{I I}$, i.e., $\delta_{I}\left(t_{c}\right)$ and $\delta_{I I}\left(t_{c}\right)$, are affected by the $\mathscr{B}_{i}$ 's computation and $\mathscr{M}$ 's communication conditions. 


$$
\begin{aligned}
\delta_{I}\left(t_{c}\right) & =\min \left(1, \frac{\mathcal{S}_{i}\left(t_{c}\right)}{\mathfrak{P}_{\mathscr{L}}}\right), \\
\delta_{I I}\left(t_{c}\right) & =1-\mathscr{W}_{\mathscr{M}}^{t_{c-1}} .
\end{aligned}
$$

Finally, at time $t_{c}$, the $A A B S$ with a given $\left(\delta_{I}\left(t_{c}\right), \delta_{I I}\left(t_{c}\right)\right) \in[0,1]^{2}$, i.e., $A A B S_{\left(\delta_{I}\left(t_{c}\right), \delta_{I I}\left(t_{c}\right)\right)}(\cdot)$, is given as follows:

$$
\begin{aligned}
\operatorname{AABS}_{\left(\delta_{I}\left(t_{c}\right), \delta_{I I}\left(t_{c}\right)\right)}\left(S^{\left.\mathfrak{P}_{\mathscr{L}}, d_{i}\left(t_{c}\right), r_{i}\left(t_{c}\right)\right)=} \max _{\lambda \in[0,1]}\left\{\lambda \mid\left(\left[\lambda \times A\left(S^{\mathfrak{P}_{\mathscr{L}}}, \mathscr{X}_{i}^{\mathscr{A}}\left(t_{c}\right)\right)\right]+\left[(1-\lambda) \times \mathscr{X}_{i}^{d}\left(t_{c}\right)\right]\right)\right\},\right. \\
\text { s.t., }\left\{\begin{array}{l}
\mathscr{X}_{i}^{\mathscr{A}}\left(t_{c}\right)=\left(\left(\delta_{I I}\left(t_{c}\right) \times r_{i}\left(t_{c}\right)\right)+\left(\left(1-\delta_{I I}\left(t_{c}\right)\right) \times d_{i}\left(t_{c}\right)\right)\right), \\
\mathscr{X}_{i}^{d}\left(t_{c}\right)=\left(\left(\delta_{I}\left(t_{c}\right) \times r_{i}\left(t_{c}\right)\right)+\left(\left(1-\delta_{I}\left(t_{c}\right)\right) \times d_{i}\left(t_{c}\right)\right)\right), \\
A A B S_{\left(\delta_{I}\left(t_{c}\right), \delta_{I I}\left(t_{c}\right)\right)}\left(S^{\mathfrak{P}_{\mathscr{L}}}, d_{i}\left(t_{c}\right), r_{i}\left(t_{c}\right)\right) \in S^{\mathfrak{P}_{\mathscr{L}}} .
\end{array}\right.
\end{aligned}
$$

Each individual $\mathbb{G}_{1 \leq i \leq n}^{I}$ game is operated independently in a parallel manner and decides each $\mathscr{B}_{i}$ 's offloading strategy $\left(\tau_{i}\right)$. Then, as game players, each $\mathscr{B}_{1 \leq i \leq n}$ participates in the $\mathbb{G}^{I I}$ game to share the $\mathfrak{M}$ for its task offloading service. Based on each WBAN's situation, the $\mathscr{B}_{i}$ 's utility function in the $\mathbb{G}^{I I}$, i.e., $\mathfrak{U}_{\mathscr{B}_{i}}(\cdot)$, is given by considering the allocated spectrum amount.

$$
\begin{aligned}
& \mathcal{U}_{\mathscr{B}_{i}}\left(\mathscr{R}_{\mathscr{B}_{i}}^{t_{c}}, \mathscr{C}_{\mathscr{B}_{i}}^{t_{c}}, N_{\mathscr{B}_{i}}, \mathfrak{M}\right)=\left(\gamma-\frac{\xi}{Y_{\mathscr{B}_{i}}}\right)^{\mathbb{C}_{i} \times Y_{\mathscr{B}_{i}}}-\mathcal{E}, \\
& \text { s.t., } Y_{\mathscr{B}_{i}}=\frac{\min \left(\mathscr{R}_{\mathscr{B}_{i}}^{t_{c}}, \mathscr{C}_{\mathscr{B}_{i}}^{t_{c}}\right)}{\mathscr{R}_{\mathscr{B}_{i}}}, \\
& \mathbb{C}_{i}=\sum_{\mathscr{T}_{\in N_{\mathscr{B}_{i}}} \frac{\mathscr{T}}{l_{M}},} \\
& \sum_{\mathscr{B}_{i} \in \mathscr{B}} \mathscr{C}_{\mathscr{B}_{i}}^{t_{c}} \leq \mathfrak{M},
\end{aligned}
$$

where $\mathscr{R}_{\mathscr{B},}^{t_{c}}$ and $\mathscr{C}_{\mathscr{B}}^{t_{c}}$ are the $\mathscr{B}_{i}$ 's requested and allocated spectrum resources, respectively, at time $t_{c} \cdot \gamma, \xi$, and $\varepsilon$ are the adjustment parameters for the $\mathfrak{U}_{\mathscr{B}_{i}}(\cdot)$. According to (7), $\mathscr{M}$ distributes the $\mathfrak{M}$ for the $\mathscr{B}_{1 \leq i \leq n}$. In the $\mathbb{G}^{I I}$ game, the individual monotonicity of each player is necessary to share the $\mathfrak{M}$. Therefore, the concept of KSBS is also adopted like the reference point decision problem. The KSBS for the $\mathbb{G}^{I I}$, i.e., $\operatorname{KSBS}_{\mathscr{M}}\left(S^{\mathfrak{M}}, d^{\mathfrak{M}}\right)$, is given by

$$
\operatorname{KSBS}_{\mathscr{M}}\left(S^{\mathfrak{M}}, d^{\mathfrak{M}}\right)=\max _{\left(Y_{\mathscr{S}_{1}}, \ldots, Y_{\mathscr{B}_{n}}\right) \in\left(S^{\mathfrak{M}}, d^{\mathfrak{M}}\right)}\left(\min _{F \in\left\{Y_{\mathscr{B}_{1}}, \ldots, Y_{\mathscr{B}_{n}}\right\}} \frac{F-d_{F}^{\mathfrak{M}}}{A_{F}\left(S^{\mathfrak{M}}, d^{\mathfrak{M}}\right)-d_{F}^{\mathfrak{M}}}\right)
$$

3.4. Main Steps of our Proposed M-W System Control Scheme. In this study, we develop a novel two-stage bargaining game model to adaptively share the limited $\mathrm{M}-\mathrm{W}$ system resource for different IoMT data services. At the first stage, multiple IoMT devices within the WBAN work together to offload their computation tasks based on the idea of $A A B S$. At the second stage, individual WBANs cooperate with each other to share the limited MEC's spectrum resource according to the idea of KSBS. Based on the real-time online monitoring, we adaptively set the bargaining factors to ensure good global properties. Therefore, our two-stage bargaining approach can achieve a mutually desirable solution while flexibly adapting the dynamic changing M-W system conditions. This feature can maximize the system efficiency while achieving the fairness of each network agent.

This study has focused on the theory based approach. Therefore, the limited resource sharing problem in the M-W platform is simply abstracted as a logical bargaining problem. To implement our approach in the real world, we simply assume that all control decisions follow the standard protocol in physical and data link layers. In addition, energy efficiency is interesting research topic in intra- and interWBAN communications. However, it is beyond the scope of this study. Therefore, there is the limitation of our scheme 
from a practical standpoint. We would like to keep the energy control issue for future research. The main steps of our proposed scheme can be described as follows:

Step 1: For our proposed scheme, the values of control parameters and adjustment factors can be found in Table 2, and the simulation scenario is given in Section 4 .

Step 2: At the time $t_{c}$, multiple IoMT devices independently generate their service requests with different types and characteristics.

Step 3: At the first stage, each individual $\mathscr{B}_{1 \leq i \leq n}$ operates its own intra-WBAN bargaining game $\left(\mathbb{G}_{i}^{I}\right)$ in a parallel fashion to select the offloading strategy $\left(\tau_{i}\right)$. Step 4: In the $\mathbb{G}_{i}^{I}$, the $\mathcal{S}_{i}^{L}\left(t_{c}\right)$ and $\mathcal{S}_{i}^{O}\left(t_{c}\right)$ are game players, and their utility functions, i.e., $U_{i}^{L}(\cdot)$ and $U_{i}^{O}(\cdot)$, are defined according to (2) and (3), respectively.

Step 5: For the $\mathbb{G}_{i}^{I}$, the concept of $A A B S$ is adopted to decide the $\tau_{i}$ value. Using (4), the $r_{i}\left(t_{c}\right)$ is obtained, and $\delta_{I}^{i}\left(t_{c}\right)$ and $\delta_{I I}\left(t_{c}\right)$ are decided according to (5). Finally, the $A A B S$ for the $\mathbb{G}_{i}^{I}$ is achieved based on (6).

Step 6: At the second stage, multiple $\mathscr{B}_{1 \leq i \leq n}$ participate in the inter-WBAN bargaining game $\left(\mathbb{G}^{I I}\right)$ to share the $\mathfrak{M}$ for their task offloading services. In the $\mathbb{G}^{I I}, \mathscr{B}_{1 \leq i \leq n}$ game players and their utility functions, i.e., $\mathfrak{U}_{\mathscr{B}_{1 \leq i \leq n}}(\cdot)$, are defined according to (7).

Step 7: For the $\mathbb{G}^{I I}$, the idea of KSBS is used to distribute the $\mathfrak{M}$ for $\mathscr{B}_{1 \leq i \leq n}$; it is obtained according to (8).

Step 8: Intra-WBAN bargaining games $\left(\mathbb{G}_{1 \leq i \leq n}^{I}\right)$ and the inter-WBAN bargaining game $\left(\mathbb{G}^{I I}\right)$ interact with each other and work together in a coordination manner.

Step 9: Constantly, each individual $\mathscr{B}_{1 \leq i \leq n}$ is selfmonitoring the current $\mathrm{M}-\mathrm{W}$ platform environments and proceeds to Step 2 for the next two-stage interactive bargaining process.

\section{Performance Evaluation}

In this section, we build a simulation environment using Matlab to evaluate the performance of our proposed algorithm. To confirm the effectiveness of our two-stage bargaining method, the simulation results are analyzed via the performance comparison with the existing TGCO, ECHM, and IDCM protocols in $[4,5,8]$. The simulation scenario and environment setup are shown as follows:

(i) The simulated $\mathrm{M}-\mathrm{W}$ platform consists of one $\mathscr{M}$ and ten WBANs where $|\mathfrak{B}|=10$.

(ii) All WBANs are located in the $\mathscr{M}$ 's covering area. Therefore, they directly communicate with the $\mathscr{M}$.

(iii) Ten IoMT devices are located in each IoMT device that generates its data applications for healthcare services.

(iv) Six different kinds of data applications are assumed based on their resource requirements, preference levels, and service duration times.
TABLE 2: System parameters used in the simulation experiments.

\begin{tabular}{|c|c|c|c|}
\hline Parameter & Value & \multicolumn{2}{|c|}{ Description } \\
\hline$n$ & 10 & \multirow{2}{*}{\multicolumn{2}{|c|}{$\begin{array}{c}\text { The total number of WBANs } \\
\text { The total spectrum capacity of MEC } \\
\text { server }\end{array}$}} \\
\hline $\mathfrak{M}$ & 5 Gbps & & \\
\hline$\eta, \theta$ & 1,1 & \multicolumn{2}{|c|}{$\begin{array}{l}\text { Adjustment parameters for the } \\
\qquad U_{i}^{L}(\cdot)\end{array}$} \\
\hline$\beta, \sigma$ & $0.5,1$ & \multirow{2}{*}{\multicolumn{2}{|c|}{$\begin{array}{l}\text { Control parameters for the } U_{i}^{O}(\cdot) \\
\text { Adjustment parameters for the } \\
\qquad \mathfrak{U}_{\mathscr{B}}(\cdot)\end{array}$}} \\
\hline$\gamma, \xi, \varepsilon$ & $1,1,1$ & & \\
\hline$l_{m}, l_{M}$ & $0.5,0.8$ & \multirow{2}{*}{\multicolumn{2}{|c|}{$\begin{array}{l}\text { The minimum and maximum } \\
\text { preference levels } \\
\text { A diminishing factor to decide } \\
\text { reference point }\end{array}$}} \\
\hline$\omega$ & 5 & & \\
\hline $\mathfrak{P}_{\mathscr{L}}$ & $1 \mathrm{GHz}$ & \multicolumn{2}{|c|}{$\begin{array}{c}\text { The total computation capacity of } \\
\qquad \mathscr{L}\end{array}$} \\
\hline Applications & $\begin{array}{l}\text { Service } \\
\text { duration }\end{array}$ & $\begin{array}{c}\text { Resource } \\
\text { requirement }\end{array}$ & $\begin{array}{l}\text { Preference } \\
\text { level }\end{array}$ \\
\hline 1 & $10 \mathrm{t}$ & $1 \mathrm{Mbps} / 1 \mathrm{MHz}$ & 0.5 \\
\hline 2 & $40 \mathrm{t}$ & $1.5 \mathrm{Mbps} / 1.5 \mathrm{MHz}$ & 0.7 \\
\hline 3 & $45 \mathrm{t}$ & $3 \mathrm{Mbps} / 3 \mathrm{MHz}$ & 0.65 \\
\hline 4 & $60 t$ & $2 \mathrm{Mbps} / 2 \mathrm{MHz}$ & 0.8 \\
\hline 5 & $90 \mathrm{t}$ & $2.5 \mathrm{Mbps} / 2.5 \mathrm{MHz}$ & 0.6 \\
\hline 6 & $35 \mathrm{t}$ & $3.5 \mathrm{Mbps} / 3.5 \mathrm{MHz}$ & 0.75 \\
\hline
\end{tabular}

margin $=" 0 p t "$ indent="0pt" style="ULC" spanacross="0" alignment $=" 0 " \quad$ keep_with_next $=" 0 " \quad$ keep_with_previous="0" justify="1" hyphenation="11"> (v) At each time epoch, the generation process for data applications is Poisson with rate $\Lambda$ (services/t), and the range of offered services was varied from 0 to 3.0.

(vi) We assume that the total computation capacity of $\mathscr{L}$, i.e., $\mathfrak{P}_{\mathscr{L}}$, is $1 \mathrm{GHz}$, and the total available spectrum amount of $\mathscr{M}$, i.e., $\mathfrak{M}$, is $5 \mathrm{Gbps}$.

(vii) To ensure the intercompatibility between computation and communication processes, we assume that one bit is equivalent to one $\mathrm{Hz}$.

(viii) The disagreement point $(d)$ in the bargaining process is set to zeros, and each time period is assumed as one second.

(ix) To reduce computation complexity, the amount of computation (or communication) allocation is specified in terms of minimum units, where one unit size is $256 \mathrm{KHz}$ (or $256 \mathrm{Kbps}$ ) in our bargaining process.

(x) System performance measures obtained on the basis of 100 simulation runs are plotted as a function of the offered data request load.

(xi) We assume the absence of physical obstacles in the wireless communications.

In Figure 3, the system throughput results of the proposed scheme and the TGCO, ECHM, and IDCM schemes are plotted based on the different service workload rates. In general, the total throughput of all schemes increases when the service rate increases, which is intuitive. It is observed that the throughput obtained using our proposed scheme is much higher than that of the other existing schemes in $[4,5,8]$, which confirms the 


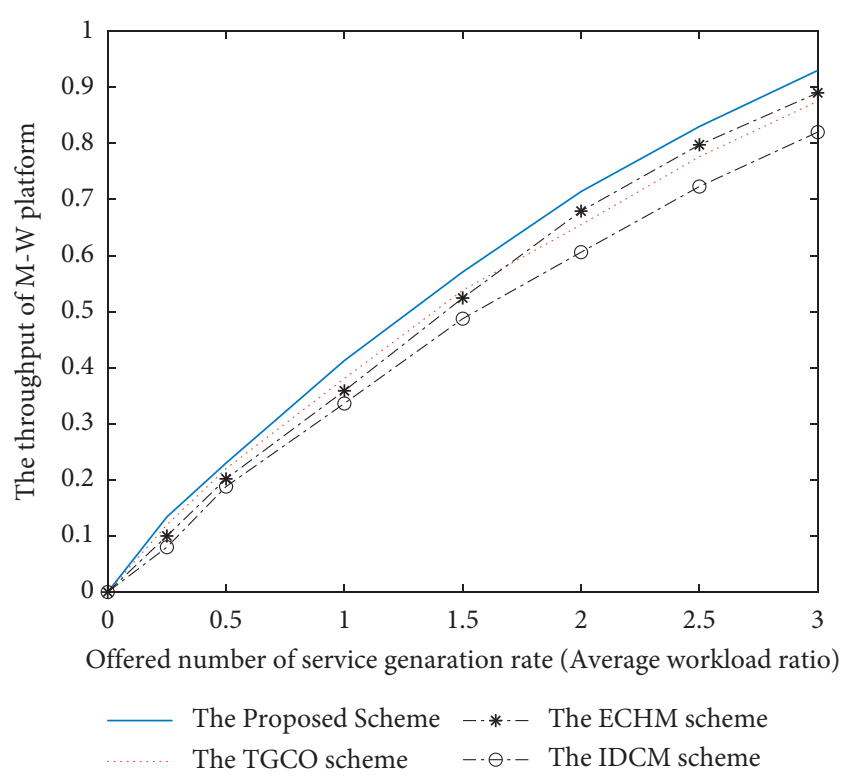

Figure 3: The throughput of M-W platform.

performance gain of our two-stage bargaining approach. The main reason is that we make bargaining decisions based on the real-time online information to adaptively approximate the optimal system throughput.

The evaluation of normalized IoMT device payoff is presented in Figure 4. As it can be seen, the proposed scheme has the highest payoff. The reason is as follows: with the increase of the workload rate, the limited $\mathrm{M}-\mathrm{W}$ platform resources in our proposed scheme are dynamically shared according to devices' preferences. Therefore, the system resource is more efficiently used while IoMT devices adjust their requests in a coordinated manner; this means that the cooperative bargaining concepts can be well applied in the resource sharing problem of the $\mathrm{M}-\mathrm{W}$ platform.

Figure 5 demonstrates the fairness among WBANs for offloading services. From the viewpoint of individual WBANs, it is another important performance criterion. Traditionally, the major challenge in developing a novel bargaining solution is to ensure a relevant tradeoff between efficiency and fairness. As mentioned earlier, the main feature of $K S B S$ is to provide a fair-efficient bargaining solution due to its individual monotonicity axiom. This implies that our proposed spectrum sharing method achieves the best fairness for different WBANs compared to the other existing TGCO, ECHM, and IDCM schemes. It is a desirable property for the real-world system operations. From the simulation results shown in Figures 3 to 5, our two-stage bargaining approach always outperforms the reference protocols. Specifically, the throughput, payoff, and fairness are improved by about $15 \%, 15 \%$, and $10 \%$, respectively. It is worth noting that our dual bargaining based strategic control mechanism contributes to the improvement of the M-W system performance.

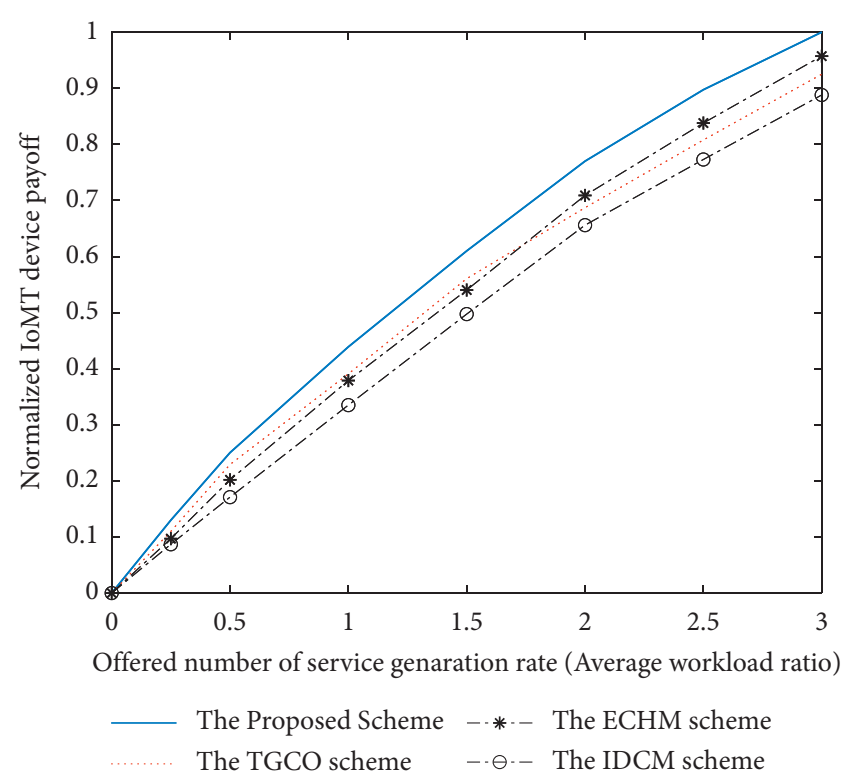

Figure 4: Normalized IoMT device payoff.

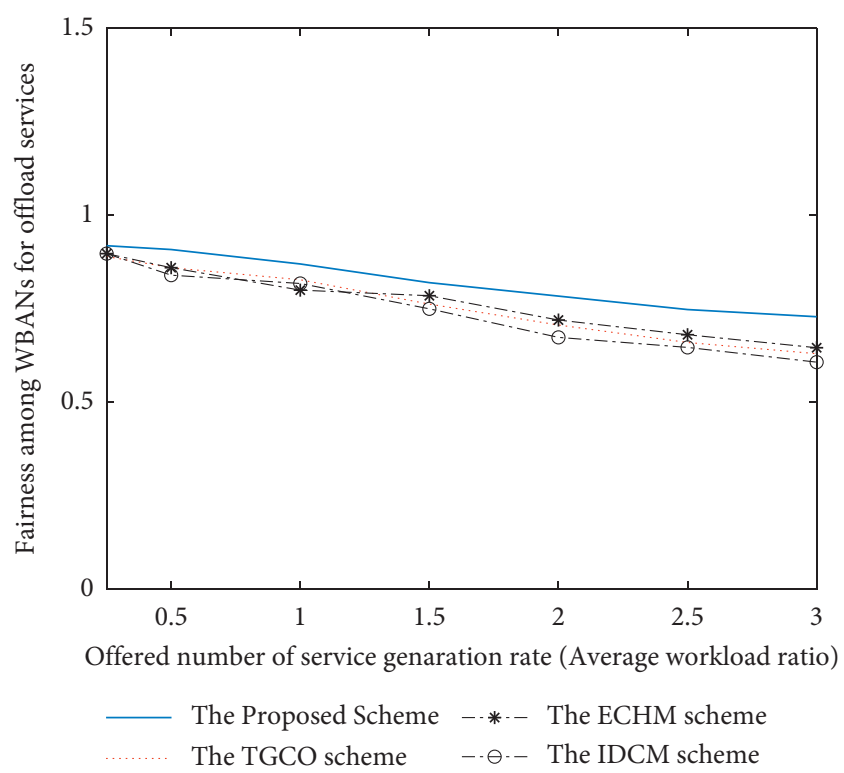

FIgUre 5: The fairness among WBANs in the M-W platform.

\section{Summary and Conclusions}

Edge computing provides a great opportunity to reduce the overhead in IoMT-based WBAN healthcare services. This paper considers a new two-stage bargaining game model for the multiple-WBAN coexisting situation. With the MEC server, each individual WBAN decides whether to perform the medical analysis task by local computing or offload it to the MEC. At the first stage, the intra-WBAN bargaining process is formulated, and the $A A B S$ is utilized to select an effective offloading strategy. At the second stage, the interWBAN bargaining process is designed, and the KSBS is adopted to share the limited MEC spectrum resource. Intraand inter-WBAN bargaining games interact sequentially to 
share the computation and communication resources of $\mathrm{M}-\mathrm{W}$ platform. With regard to the real-time measured bargaining factors, we can be more responsive to current system conditions. Therefore, our proposed method has achieved greater and reciprocal advantages under dynamically changeable $\mathrm{M}-\mathrm{W}$ system situations. Performance evaluations confirm the effectiveness of our two-stage bargaining approach compared with the existing TGCO, $E C H M$, and IDCM protocols.

Currently, in this work, we assume a simple one-hop topology for WBANs. Therefore, we did not consider any routing issues. Therefore, the future extension of this work can include the routing concept for inter-WBAN communications. In addition to this, we plan to extend this work by incorporating energy control issue to maximize the service satisfaction with necessary modifications in the bargaining procedure. Additionally, we envision implementing a social choice theory in order to achieve the collective decision of different WBANs with the MEC server.

\section{Data Availability}

The data used to support the findings of this study are available from the author upon request (swkim01@sogang.ac.kr).mailto:swkim01@sogang.ac.kr

\section{Conflicts of Interest}

The author declares that there are no conflicts of interest regarding the publication of this paper.

\section{Authors' Contributions}

Sungwook Kim is the sole author of this work, and ES participated in the design of the study and performed the statistical analysis.

\section{Acknowledgments}

This research was supported by the MSIT (Ministry of Science and ICT), Korea, under the ITRC (Information Technology Research Center) support program (IITP-20212018-0-01799) supervised by the IITP (Institute for Information \& Communications Technology Planning \& Evaluation) and was supported by the Basic Science Research Program through the National Research Foundation of Korea (NRF) funded by the Ministry of Education (No. 2021R1F1A1045472).

\section{References}

[1] J. Pan and J. McElhannon, "Future edge cloud and edge computing for internet of things applications," IEEE Internet of Things Journal, vol. 5, no. 1, pp. 439-449, 2018.

[2] Z. Liu, B. Liu, and C. W. Chen, "Transmission-rate-adaption assisted energy-efficient resource allocation with QoS Support in WBANs," IEEE Sensors Journal, vol. 17, no. 17, pp. 5767-5780, 2017.

[3] S. Misra and S. Sarkar, "Priority-based time-slot allocation in wireless body area networks during medical emergency situations: an evolutionary game-theoretic perspective," IEEE
Journal of Biomedical and Health Informatics, vol. 19, no. 2, pp. 541-548, 2015.

[4] X. Yuan, H. Tian, H. Wang, H. Su, J. Liu, and A. Taherkordi, "Edge-enabled wbans for efficient QoS provisioning healthcare monitoring: a two-stage potential game-based computation offloading strategy," IEEE Access, vol. 8, pp. 92718-92730, 2020.

[5] Z. Ning, P. Dong, X. Wang et al., "Mobile edge computing enabled $5 \mathrm{G}$ health monitoring for internet of medical things: a decentralized game theoretic approach," IEEE Journal on Selected Areas in Communications, vol. 39, no. 2, pp. 463-478, 2021.

[6] M. Cicioğlu and Ç. Ali, "Energy efficiency solutions for IEEE 802.15. 6 based wireless body sensor networks," Wireless Personal Communications, vol. 199, pp. 1-15, 2021.

[7] M. Cicioğlu and Ç. Ali, "Energy-efficient and SDN-enabled routing algorithm for wireless body area networks," Computer Communications, vol. 160, pp. 228-239, 2020.

[8] B. Zhang and Y. Zhang, "An individual differentiated coexisting mechanism for multiple wireless body area networks based on game theory," IEEE Access, vol. 6, pp. 54564-54581, 2018.

[9] S. Kim, Game Theory Applications in Network Design, IGI Global, Hershey, PA, USA, 2014.

[10] C. Alós-Ferrer, J. García-Segarra, and M. Ginés-Vilar, “Anchoring on Utopia: a generalization of the kalai-smorodinsky solution," Economic Theory Bulletin, vol. 6, no. 2, pp. 141-155, 2018.

[11] E. Karagozoglu, K. Keskin, and E. Ozcan-Tok, "Between Anchors and aspirations: a new family of bargaining solutions," Review of Economic Design, vol. 23, no. 1-2, pp. 53-73, 2019.

[12] S. Kim, "New bargaining game based computation offloading scheme for flying ad-hoc networks," IEEE Access, vol. 7, pp. 147038-147047, 2019.

[13] S. Amit, S. Bera, and S. Misra, "Link-quality-aware resource allocation with load balance in wireless body area networks," IEEE Systems Journal, vol. 12, no. 1, pp. 74-81, 2018.

[14] S. Kim, "Bargaining Solution-based resource allocation scheme for cloud-assisted wireless body area networks," Wireless Communications and Mobile Computing, vol. 2020, Article ID 5470428, 10 pages, 2020. 